\title{
Assessment of health needs in multidisciplinary care
}

\section{A Young}

Comprehensive care for people with disabling neurological disease-finding the best approach

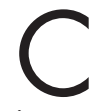
omprehensive care for people with disabling neurological illnesses is widely acknowledged to require input from many specialisms. The foundation of such multifaceted care should be a detailed assessment of physical and psychosocial function and health needs, ideally by experienced individuals able to work in a transdisciplinary way and possessing excellent communication skills. Such individuals would be capable of both identifying a clinical problem, such as impaired heel strike, and determining the most appropriate treatment, such as stretches from the physiotherapist, botulinum toxin from the physician or orthopaedic release. Clearly clinicians with the wide training and experience for such global assessment are in short supply. In their absence, other approaches to identifying health needs for multidisciplinary care have been assessed. Asking each discipline to independently assess the patient in case his or her input could be relevant is a poor use of resources and burdensome to the patient. Being seen simultaneously by a wide range of rehabilitation professionals was the least popular format for a multidisciplinary neurological clinic. ${ }^{1}$ So if individualised assessment is not available to all, and the unfocused approach is unpopular and wasteful, can an instrument be used to guide assessment? In the paper by Hoogervorst et al (this issue, 20-24) the utility of the INTERMED in identifying multidisciplinary care needs in people with multiple sclerosis was assessed. This instrument involves a single professional observer conducting a structured interview across biological, psychological, social, and health care domains.

Validation of assessment instruments that encompass the spectrum of health needs is an important area for future research. The possibility of a clinician using a single instrument to assess several health domains offers clear advantages with regard to time, intrusiveness to the patient, and integration of a care plan. However, it will be important to research the relationship between the health needs indicated by such assessments and the patients' perception of their health needs. There is often poor concordance between health needs identified by disabled people compared with professionals involved in their care. ${ }^{3}$

There are several interesting methodological approaches to be explored in striving to increase the accuracy and completeness of instruments aiming to assess patients' function and health needs. Firstly, the use of patient selfreport measures would be efficient and address some concerns over the mismatch of perceptions between professional and patient. For example, it may be that patient self-report could be used to assess disability in multiple sclerosis. ${ }^{4}$
Secondly, the sophistication of audioassisted self-interviewing may allow information to be obtained without a clinician needing to administer the assessment. Indeed, randomised controlled trials suggest potentially sensitive questions may be answered more honestly by computer-assisted interviewing. ${ }^{5}$

Improving assessment of health needs is futile if needs are not to be met. Underpinning research to improve assessment therefore must be a commitment to provide services. Those organising, and funding, multiple sclerosis care might reflect on the health needs identified in the 100 consecutive new patients with multiple sclerosis referred to neurology outpatients who underwent a detailed assessment (for the INTERMED study), whether or not they appeared to need multidisciplinary care. For $61 \%$, another discipline besides the neurologist and multiple sclerosis nurses was needed.

J Neurol Neurosurg Psychiatry 2003;74:5

\section{Author's affiliation}

Walton Centre for Neurology \& Neurosurgery, Lower Lane, Fazakerley, Liverpool L9 7L

Correspondence to: C A Young; carolyn.young@thewaltoncentre.nhs.uk

\section{REFERENCES}

1 Barnes MP, Skeil DA. Outpatients in neurological rehabilitation. Int J Rehabil Res 1996:19;39-45.

2 Hoogervorst ELJ, de Jonge $P$, Jelles B, et al. The INTERMED: a screening instrument to identify multiple sclerosis patients in need of multidisciplinary treatment. J Neurol Neurosurg Psychiatry 2002;74:20-4.

3 Kersten P, George S, McLellan L, et al. Disabled people and professionals differ in their perceptions of rehabilitation needs. J Public Health Med 2000:22;393-9.

4 Solari A, Amato MP, Bergamaschi R, et al. Accuracy of self-assessment of the minimal record of disability in patients with multiple sclerosis. Acta Neurol Scand 1993:87;43-6

5 Metzger DS, Koblin B, Turner C, et al. Randomized controlled trial of audio computer-assisted self-interviewing: utility and acceptability in longitudinal studies. HIVNET Vaccine Preparedness Study Protocol Team. Am J Epidemiol 2000:152;99-106. 


\section{Blood-brain barrier permeability in type II diabetes}

\section{J V Bowler}

\section{There is a direct link between diabetes, a leaky blood-brain barrier, leukoaraiosis, and cognitive impairment}

$\mathrm{D}$ iabetes has an important and readily demonstrable association with cognitive impairment and stroke. Starr et al, in their paper on page 000, have concentrated on a more subtle pathology, that of leukoaraiosis. They have shown increased blood-brain barrier permeability in well controlled diabetic patients and in association with leukoaraiosis in both patients and controls. The authors also draw attention to the poorly recognised association between diabetes, impaired cognition, and leukoaraiosis. When leukoaraiosis was first seen on CT and later on MRI it was routinely dismissed as unimportant. However, leukoaraiosis is associated with cognitive impairment and increases the risk of dementia when seen in conjunction with other dementing illnesses, whether these be primarily degenerative such as Alzheimer's disease, or vascular. ${ }^{2}$ Its influence is often subtle in individuals, but in populations it makes a significant contribution to cognitive impairment.

While hypertension is the most important risk factor for leukoaraiosis, diabetes is also well recognised. Importantly, impairment of glucose tolerance in mid-life correlates with later life leukoaraiosis, ${ }^{3}$ suggesting an opportunity for prevention.

There is also a direct association between diabetes and cognitive impairment of various kinds in much the same way that leukoaraiosis is associated with dementia, except that the association is weaker, as would be expected in view of the role of hypertension. ${ }^{4}$ This association is not mediated by frank infarction and is unlikely to be through acceleration of the various primary processes underlying the degenerative dementias.

What then is the mechanism? Endothelial damage and the development of lipohyalinosis and subsequent lacunar infarction play a part. However, leukoaraiosis also has a role and does not include frank infarction. The authors draw attention to "ischaemic demyelination," but a related and much more general concept was originally proposed by Brun and Englund and termed "incomplete infarction". ${ }^{5}$ It is characterised by demyelination, loss of axons and oligodendroglia, and mild reactive gliosis without cavitation, and is thought to be ischaemic in origin. The mechanism is not considered to be chronic low perfusion, but rather diminished perfusion reserve with episodic mild ischaemia occurring as a result of intermittent changes in cerebral perfusion pressure. Cerebral blood flow in affected areas lies within normal limits at rest. However, in areas of leukoaraiosis other changes are seen which at their mildest may consist just of local oedema. ${ }^{6}$ Extensive areas of such changes may be seen without local evidence of completed ischaemic events, leading to the suggestion of leakage of serum proteins, which are known to be toxic; it is here that increased bloodbrain barrier permeability may have a direct and important action. All of these pathological changes appear the same on standard MRI sequences, resulting in the not uncommon experience of patients with extensive leukoaraiosis without clinical or cognitive deficits on the one hand, through to patients with relatively mild leukoaraiosis but with significant deficits on the other.

There is therefore a direct link between diabetes, a leaky blood-brain barrier, leukoaraiosis, cognitive impairment, and-in association with other dementing illnesses-an exacerbation of dementia. In individual cases this effect is slight, but with the increasing numbers of cognitively impaired elderly people it becomes important, not least because the process is modifiable by tight control of diabetes and by meticulous control of any coexistent hypertension with ACE inhibitors.

J Neurol Neurosurg Psychiatry 2003;74:6

\section{Author's affiliation}

Dr J V Bowler, Department of Neurology, Royal Free Hospital, London NW3 2QG

Correspondence to: J V Bowler;

j.bowler@rfc.ucl.ac.uk

\section{REFERENCES}

1 Starr JM, Wardlaw J, Ferguson K, et al. Increased blood-brain barrier permeability in type II diabetes demonstrated by gadolinium magnetic resonance imaging. J Neurol Neurosurg Psychiatry 2003;74:70-76

2 Breteler MM, van Amerongen NM, van Swieten JC, et al. Cognitive correlates of ventricular enlargement and cerebral white matter lesions on magnetic resonance imaging. The Rotterdam Study. Stroke imaging. The Rotter

3 Carmelli D, Swan GE, Reed T, et al. Midlife cardiovascular risk factors and brain morphology in identical older male twins. Neurology 1999;52: 111 19-24.

4 Knopman D, Boland LL, Mosley T, et al. Cardiovascular risk factors and cognitive decline in middle-aged adults. Neurology 2001;56:42-8.

5 Brun A, Englund E. A white matter disorder in dementia of the Alzheimer type: a pathoanatomical study. Ann Neurol 1986;19:253-62.

6 Munoz DG, Hastak SM, Harper B, et al. Pathologic correlates of increased signals of the centrum ovale on magnetic resonance imaging. Arch Neurol 1993;50:492-7. 